\title{
LATE BLIGHT SEGMENTATION IN TOMATOES USING SPATIAL DOMAIN METHODS AND COLOUR ANALYSIS
}

\section{Agricultural Science}

I. Galvan-Torres

\author{
A.S. Cortés- \\ González \\ C.N. López-Mejía
}

Instituto Politécnico Nacional. Escuela Superior de Cómputo. Av. Juan de Dios Batíz, esq. Miguel Othón de Mendizabal, Mexico city 0773, Mexico.

Instituto Politécnico Nacional. Escuela Superior de Cómputo. Av. Juan de Dios Batíz, esq. Miguel Othón de Mendizabal, Mexico city 0773, Mexico.

\section{B. Luna-Benoso}

\section{J.C. Martínez- Perales*}

Instituto Politécnico Nacional. Escuela Superior de Cómputo. Av. Juan de Dios Batíz, esq. Miguel Othón de Mendizabal, Mexico city 0773, Mexico.

Instituto Politécnico Nacional. Escuela Superior de Cómputo. Av. Juan de Dios Batíz, esq. Miguel Othón de Mendizabal, Mexico city 0773, Mexico.

Instituto Politécnico Nacional. Escuela Superior de Cómputo. Av. Juan de Dios Batíz, esq. Miguel Othón de Mendizabal, Mexico city 0773, Mexico. ${ }^{*}$ Corresponding Author

\section{ABSTRACT}

Agricultural productivity is an important factor in a country's economic development. Therefore, the diagnosis of plant diseases is a field of research of great importance for the agricultural sector since it allows us to help recommend strategies to prevent the spread of diseases, thus reducing economic losses. Currently, with the rise of computer systems, computer systems have been developed that allow computer assisted diagnosis in different fields of research, including the agricultural sector. Since late blight is one of the main diseases due to its high incidence and severity, this paper proposes a methodology to obtain late blight segmentation in tomato leaf images through image analysis and color analysis using the HSV color model. The proposed methodology was applied to a set of publicly available PlantVillage images, to which late blight segmentation was obtained.

\section{KEYWORDS}

segmentation, tomatoes, spatial domain, colour analysis

\section{I.INTRODUCTION}

According to the Food and Agriculture Organization (FAO), there is a need to increase global food production by 70 to be able to provide food for the 9.1 billion people projected for 2050 , this will be achieved by boosting the transformation of food production with the integration of new technologies. According to FAO data, the surface area of tomatoes worldwide grew at an average annual rate of 1.4 between 2007 and 2017, to be located in 4.8 million hectares. Of the total area harvested in 2017, this is concentrated in 5 countries: China with 21.2India with 16.4Nigeria with 12.2Turkey with 3.9 and Egypt with $3.8[1,2]$. Statistics to the National Institute of Food and Agriculture (U.S.A.), worldwide crop losses between 40 and 50 due to pests and different crop diseases, so monitoring and detection of plant diseases is of utmost importance for sustainable agricultural development [3] The detection of plant diseases through manual monitoring is a slow work that requires the preparation of an expert in the agricultural area. However, with the rise of computer systems development, and mainly in the development of the field of automatic learning, works have been developed that allow the detection of diseases in plants in an automatic way $[4,5,6]$. One of the first tasks of the computer systems that make use of automatic learning applied to problems involving images, is to segment the lesions present in the images and that are desired to classify. Segmentation of lesions in leaf images serves as an important tool in the evaluation and diagnosis of plant diseases. Segmentation is the process of isolating the objects that make up an image, that is, the partitioning of the image into disjointed regions, in such a way that each region is homogeneous with respect to certain properties, such as levels of gray, contrast, texture, etc. [7,8].

One of the main diseases due to its high incidence and severity is late blight. Late blight is caused by the Oomycete Phytophthora Infestans (Mont.) de Bary and is considered one of the most devastating diseases due to the destruction caused to tomato and potato crops, since in humid environments it can lead to the complete loss of the crop in a few days $[9,10]$. Several works have been developed with different approaches that allow the detection of late blight in tomato plants from leaf images. The first task of each of these works is to segment lesions on the leaves to later classify them. Singh et al. [11] propose a methodology based on deep learning for the phenotyping of plant stress, for the part of segmentation of lesions on the leaves of the plants, they make use of characteristics map and in this way they highlight the area of the diseased leaf. Sabrol et al [12] use Otsu's method for the segmentation module from which they extract a total of 24 characteristics such as colour, shape and texture among others to feed their classification tree. Vianna et al [13] estimate the degree of late blight infestation in tomato leaves, and for the segmentation of leaf lesions, they use the color tone of the individual pixels of each of the leaves by means of the RGB model, to which they add the values of the HSL model to each pixel in order to later perform the recognition task. Arakeri et al. [14] analyze the late blight disease in tomato leaves, the segmentation of the leaves and the lesions are carried out by thresholding, from which they then feed the k-means classifier. Sharma et al. [15] manually segment lesions in plants to feed their convolutional neural network classifier. Singh et al. [16] propose a methodology that allows early detection of plant diseases, for the segmentation stage they use the LabelImg tool [17], a tool that allows the manual creation of delimiting tables around the leaves.

This paper proposes a methodology to segment late blight lesions by analyzing tomato images. For this purpose, color analysis in the HSV color model is proposed. The document is organized in the following way: section 1 shows the introduction, section 2 presents the tools necessary for the development of the work, section 3 shows the proposed methodology, section 4 presents the experiments and results, and section 5 shows the conclusions.

\section{MATERIALS AND METHODS \\ Digital Imaging}

A digital image is composed of image elements called pixels. Usually, the pixels are arranged in an ordered rectangular matrix. The size of an image is determined by the dimensions of this pixel array. The width of the image is the number of columns $W$ and the height of the image is the number of rows $H$ of the matrix. Therefore, the pixel matrix is a matrix of size $X W$. To refer to a specific pixel within the matrix of an image, we will do so by means of its coordinate $(x, y)$. In addition, each pixel position $(x, y)$ has an associated intensity of color that we will denote as $I(x, y)$. Figure 1 shows the concept of a digital image.

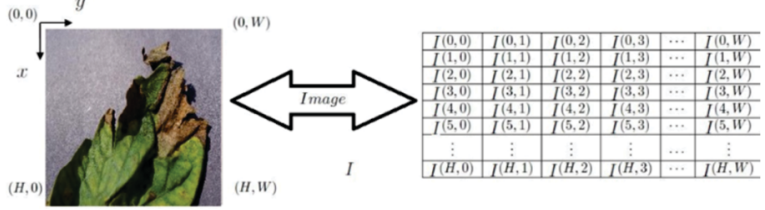

Fig.1: Digital image 


\section{Color models}

The handling of color pixels is of utmost importance in image processing, due to the characteristics it allows to stand out in the image, such as brightness, chromaticity, intensity of a certain color, etc. There are different color models, one of the most common is the RGB (RedGreen-Blue) model. The RGB model is based on the Cartesian coordinate system and is a tetrahedron-shaped color space, where the values of each RGB component are found in three vertices: cyan, magenta and yellow, with black being the color that corresponds to the origin and white the most alleged of these. On the other hand, the HSV (Hue-Saturation-Value) model uses a prism to locate the position of each of the components that make up the image. The $\mathrm{H}$ component is the circular region with values between $0^{\wedge} \mathrm{o}$ y $360^{\wedge} \mathrm{o}$, the $\mathrm{S}$ component is located on the horizontal axis of the cone and the $\mathrm{V}$ component on the vertical axis. See figure 2 .

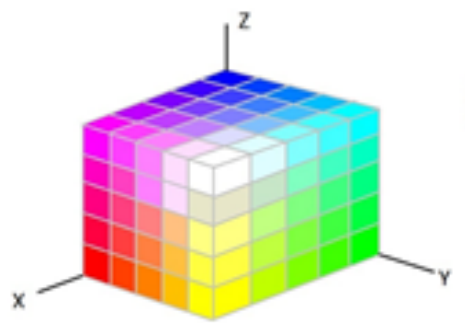

a)

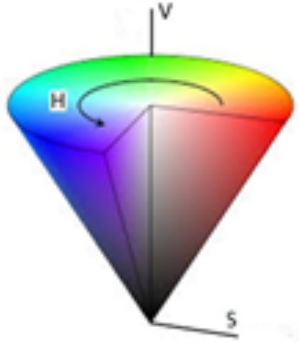

b)
Fig. 2: Colour model, in a) RGB, and in b) HSV.

\section{Image Histogram}

The histogram of a digital image with gray levels in the range $[0,255]$ is a discrete function Histo $[k]$ which represents the number of colors for each level of gray $(k=0, \ldots, 255)$. The graphical representation of this function for all $\mathrm{k}$ provides an overall description of the image's appearance [18]. Although the histogram does not indicate anything specific about the image content, it provides us with very useful information about the possibility of highlighting interesting features of the image.

\section{Otsu's method}

Otsu's method consists of splitting an image into grey levels with $\mathrm{N}$ pixels and $\mathrm{L}$ possible different levels. The probability of occurrence of the grey level i in the picture is given by $p_{i}=\frac{f_{i}}{N}$ with $f i$ the frequency of repetition of the i-th ( $I$ $=1,2, \ldots, L)$ grey level. Consider $\omega_{1}=\sum_{i=1}^{t} p_{i}$ and $\omega_{2}=\sum_{i=t+1}^{L} p_{i}$ where $1,2, \ldots, t$ and $t+1, t+2, \ldots, L$, are the grey levels corresponding to the classes $C_{1}$ and $C_{2}$ (binaryization), then the probability distributions of the classes $C_{1}$ and $C_{2}$ are given by $\frac{p_{1}}{\omega_{1}(t)}, \frac{p_{2}}{\omega_{1}(t)}, \ldots, \frac{p_{t}}{\omega_{1}(t)}$ and $\frac{p_{t+1}}{\omega_{2}(t)}, \frac{p_{t+2}}{\omega_{2}(t)}, \ldots, \frac{p_{L}}{\omega_{2}(t)}$ respectively. The averages for each class respectively are defined as $\mu_{1}=\sum_{i=1}^{t} \frac{i P_{i}}{\omega_{1}(t)}$ and $\mu_{2}=$ $\sum_{i=t+1}^{L} \frac{i P_{i}}{\omega_{2}(t)}$ with a variance between classes of a threshold image is given by $\sigma_{B}^{2}=\omega_{1}\left(\mu_{1}-\mu_{T}\right)^{2}+\omega_{2}\left(\mu_{2}-\mu_{T}\right)^{2}$. The optimal threshold that maximizes variance is $t^{*}=\operatorname{Max}_{t}\left\{\sigma_{B}^{2}(t)\right\}$

\section{Related Components}

A related component essentially consists of grouping pixels from the same region by assigning them the same label. To clarify the definition of a related component, let us consider 8 -connectivity. It is said that a pixel $p$ is connected by the 8-pixel $q$ connectivity If $p$ is one of its eight neighbors adjacent to $q$ (Figure 3). A one-pixel $p$ path to a pixel $q$ is a sequence of pixels $r_{0}, r_{1}, \ldots, r_{k}$ such that $r_{0}=p, r_{k}=q$ and the pixel $r_{i}$ is connected by the 8-pixel connectivity $r_{i+1}$ for each $0 \leq i \leq k$. A region $S$ of an image is a related component if for each pair of pixels $p, q$ at $S$. There is a way from $p$ to $q$ where every pixel that defines the path is also in $S$.

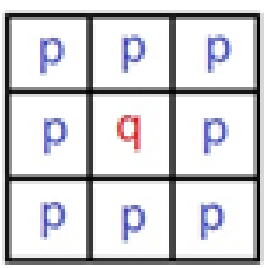

Fig. 3: 8-connectivity. 3. Proposed Model

This section presents the proposed methodology that allows the segmentation of late blight lesions in red tomato leaves by means of digital images. Let us consider a color image as shown in figure 1. First, the RGB color model was considered, and the average over the color intensity of each of the $\mathrm{R}, \mathrm{G}$ and B planes was used, obtaining $(x, y)=$ $(\mathrm{R}(x, y)+\mathrm{G}(x, y)+\mathrm{B}(x, y)) / 3$ for each pixel coordinate to for each pixel coordinate to obtain the grayscale image. Figure $4 \mathrm{~b}$ ) result of obtaining a grayscale image, and on it, the negative operator was applied $\operatorname{Neg}(x, y)=255-\mathrm{I}(x, y)$. A media filter with a mask of $3 \times 3$. Figure 4 shows the result of converting a color image to grayscale, then the negative of the image and finally the result of applying the media filter.

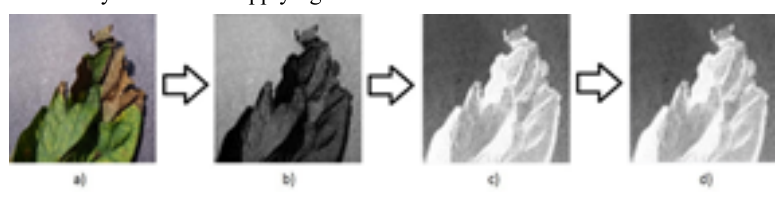

Fig. 4: Image in a) color, b) grayscale, c) negative of the image and d) with media filter

Once an image like the one shown in Figure 4-d) was obtained, the Otsu method was used to obtain the optimal binarization threshold, the threshold at which the image was binarized. Figure 5 shows the result of applying the Otsu method.
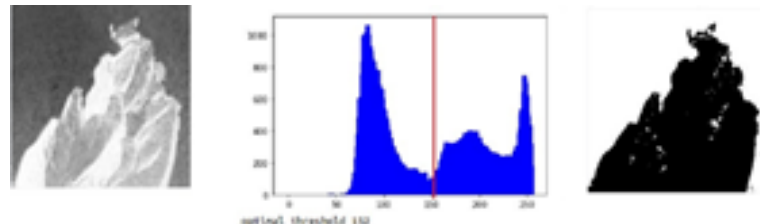

\section{Fig. 5: Binarization by Otsu}

Once the Otsu method was applied, the segmentation of the sheet was carried out in the following way: the connected components were found by means of the 8-connectivity (figure 6-a), and the connected component of greater area was eliminated, in this case, the component of greater area is represented by means of the color yellow, to eliminate the noise, those connected components are eliminated that are not delimited by means of an area of black pixels, the result is an image like the one shown in figure 6-c). Figure 6-d) shows the result of the color sheet segmentation.

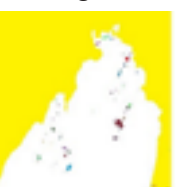

a)

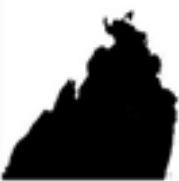

b)

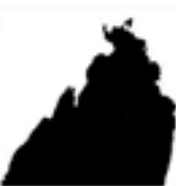

c)

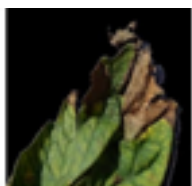

ब)
Fig. 6: Segmentation of the tomato leaf

Figure 7-a) shows the result after applying the Otsu method to another image of tomato leaf. In this case, it can be seen that there is a related component marked in orange that is present after the larger area related component has been removed as shown in Figure 7-b) and 7-c). However, since this related component is not delimited by an area of black pixels, it is removed as with the noise in figure 6-b), leaving finally the image of the segmented sheet as shown in figure 7-d).

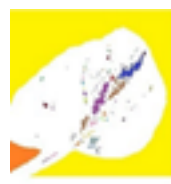

*)

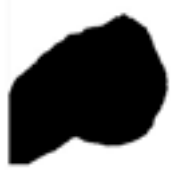

b)

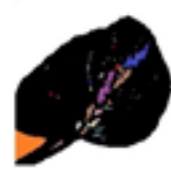

c)

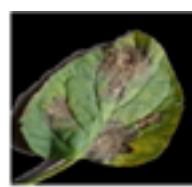

\section{Fig. 7: Segmentation of the tomato leaf}

Once the image is segmented, the color model is changed from RGB to HSV. The HSV model is designed to align more closely with the way human vision perceives color creation attributes. HSV ranges from $0^{\circ}$ $360^{\circ}$. A tone of $0^{\circ}$ is red, from $120^{\circ}$ is green, from $240^{\wedge} 0$ is blue, etc. as shown in Figure 8 .

Fig. 8: Colour spectrum HSV.

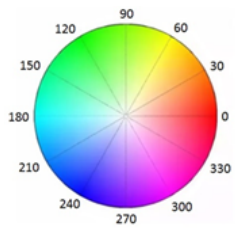


The HSV model was used to segment late blight-type leaf lesions. The color analysis of the pixels in the tomato leaf varies according to the disease, and in the case of late blight the table shown in Figure 9 shows the color thresholds at which a plant is damaged by this disease and the thresholds at which it is healthy, the values shown in the table were validated by experts in the area of Phytopathology.

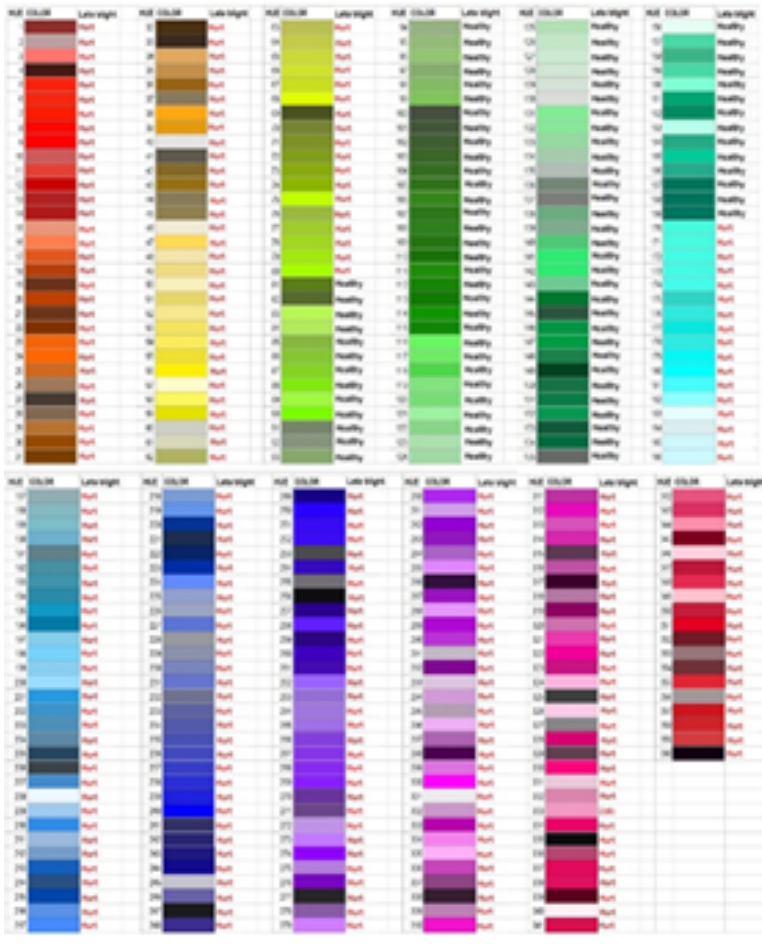

Fig. 9: Validated green and yellow colour range.

Using threshold segmentation on the Hue component of the HSV color model, and making use of the thresholds for which the tomato leaf is diseased shown in the table in Figure 9, late blight lesions were segmented on the segmented leaves. Figure 10 shows the final result of the late blight segmentation of the image in figure 1, to which each step of the methodology was applied. a)

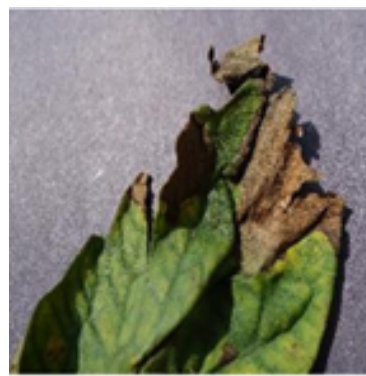

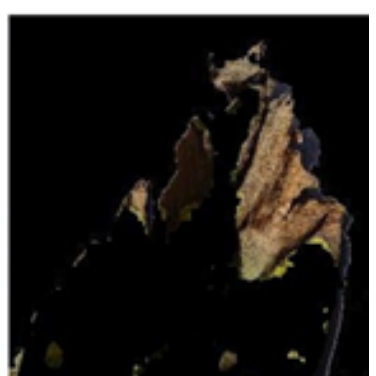

b)
Fig. 10: Segmentation of late blight in tomato leaves.

\section{Experiments and Results}

In this work we used the PlantVillage image bank [19]. PlantVillage is an open access repository of more than 70,000 images of different diseases in different types of plants, of which, it is made up of 1000 images showing late blight disease in tomato leaves. For the most part, the images were captured by several cell phone cameras in a controlled environment with a resolution of 0.1 megapixels [20] The methodology described in section 3 was applied to each of the images. Given a color image from the PlantVillage repository, the RGB model was first used, and by averaging the three channels R, G and B, it was converted to grayscale. $3 \times 3$. The image was binary and the associated negative components of the Otsu-threshold image were calculated. The largest associated component and those associated components that were not bounded by an area of black pixels obtained by Otsu binary were removed. In this way, the segmented leaf area was obtained. Once the colored leaf was segmented, the HSV color model was considered, and on the HSV model it was segmented by thresholding on the $\mathrm{H}$ component, considering the values of the table shown in figure 9. Figure 11 shows the result of applying the methodology to four plants in the PlantVillage repository.

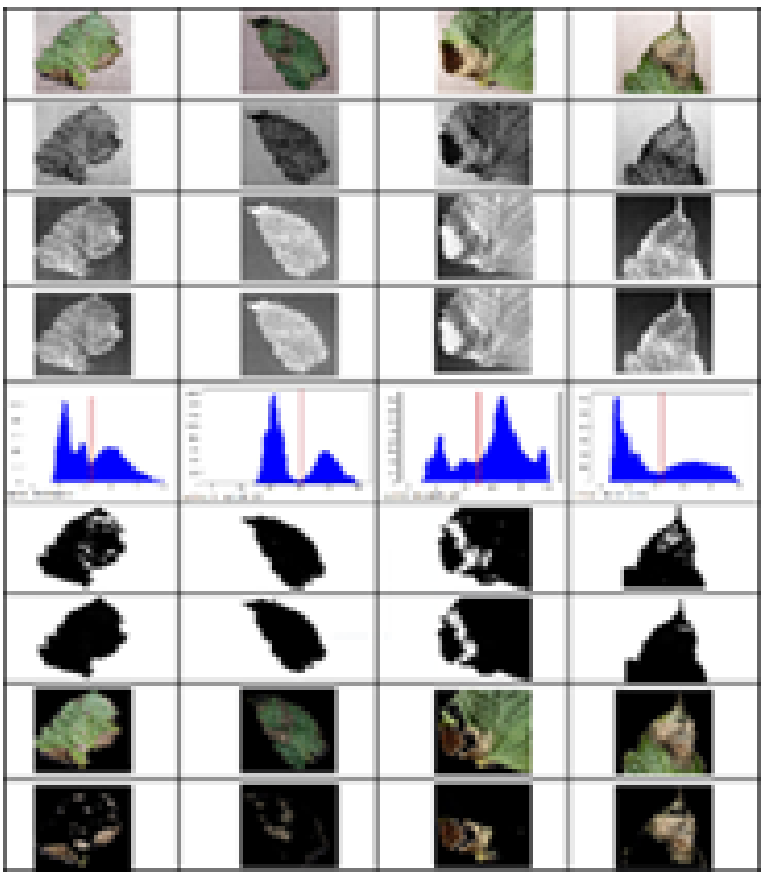

Fig. 11: Segmentation of late blight to different tomato images

\section{CONCLUSIONS}

In this work, a methodology was presented that allows the segmentation of late blight disease present in tomato leaves. Late blight is one of the main leaf diseases in crops because of its high incidence and severity with which it attacks the plants. In this work we used the PlantVillage image bank of free access, which contains 1000 images of tomato leaves affected by late blight. The proposed methodology was applied to each of the images, which consisted of first obtaining the grayscale image from the color image, for which it was converted to grayscale by averaging the three RGB channels. $3 \times 3$ The image was then converted to the HSV model, where it was segmented by thresholding from the color analysis of pixels in which late blight is present as shown in the table in Figure 9, values that were validated by experts in the area of Phytopathology. Table 11 shows the result of applying the methodology to 4 images from the PlantVillage image bank. It can be seen that the methodology manages to segment the damaged area.

\section{ACKNOWLEDGEMENTS.}

The authors would like to thank the Instituto Politécnico Nacional (Secretaría Académica, COFAA, EDD, SIP and ESCOM) for their economical support to develop this work.

\section{REFERENCES:}

[1] Food and Agriculture Organization(FAO). http://www.fao.org/home/en

[2] Fideicomisos Instituidos en Relaci'on con la Agricultura(FIRA) http://www.fira.gob.mx

[3] S. Baranwal, S. Khandelwal, A. Arora. Deep Learning Convolutional Neural Network for Apple Leaves Disease Detection. International Conference on Sustainable Computing in Science, Technology and Management (SUSCOM-2019). Amity University Rajasthan, Jaipur, India.

[4] M. Arsenovic, M. Karanovic, S. Sladojevic, A. Anderla, D. Stefanovic. Solving Curren Limitations of Deep Learning Based Approaches for Plant Disease Detection. Symmetry 2019,11,939; doi:10.3390/sym11070939

[5] Konstantinos P. Ferentinos. Deep learning models for plant disease detection and diagnosis Computers and Electronics in Agriculture, Volume 145, February 2018, Pages $311-318$

[6] Haseeb Nazki, Sook Yoon, Alvaro Fuentes, Dong Sun Park. Unsupervised image translation using adversarial networks for improved plant disease recognition Computers and Electronics in Agriculture, Volume 168, January 2020, Article 105117

[7] Chafik Djalal Kermad, Kacem Chehdi. (2002). Automatic image segmentation system through iterative edgeregion co-operation. Image and Vision Computing, Volume 20 Issue 8, Pages 541555 .

[8] Hai Gao, Weisi Lin, Ping Xue, Wan-Chi Siu. (2006). Marker-based image segmentation relying on disjoint set union. Signal Processing: Image Communication, Volume 21 , ssue 2, Pages 100-112

[9] T. Mulugeta, K. Abreha, H. Tekie, B. Mulatu, M. Yesuf, E. Andreasson, E. Liljeroth, E. Alexandersson. Phosphite protects against potato and tomato late blight in tropical climates and has varying toxicity depending on the Phytophthora infestans isolate. Crop Protection, Volume 121, July 2019, Pages 139-146

[10] Daniel A. C. Lage, Waldir A. Marouelli, Adalberto C. Caf'e-Filho. Management of 
powdery mildew and behaviour of late blight under different irrigation configurations in organic tomato Crop Protection, Volume 125, November 2019, Article 104886

[11] A. Singh, B. Ganapathysubramanian, S. Sarkar,A. Singh. (2018). Deep Learning for Plant Stress Phenotyping: Trends and Future Perspectives. Trends in Plant Science. 23. 10.1016/j.tplants.2018.07.004.

[12] H. Sabrol, K. Satish. Tomato Plan Disease Classification in Digital Images using Classification Tree. International Conference on Communication and Signal Processing, April 6-8, 2016, India.

[13] G. K. Vianna, G. V. Cunha, G. S. Oliveira.A Neural Network Classifier for Estimation of the Degree of infestation by Late Blight on Tomato Leaves. World Academy of Science, Engineering and Technology. Imnternational Journal of Computer and Information Engineering. Vol 11, No. 1,2017.

[14] M.P.Arakeri, M. Arun, R. K. Padmini. Analysis of late Blight disease in Tomato Leaf Using Image Processing Techniques. I. J. Engineering and Manufacturing, 2015, 4, 12-22.

[15] Parul Sharma, Yash Paul Singh Berwal, Wiqas Ghai. Performance analysis of deep learning $\mathrm{CNN}$ models for disease detection in plants using image segmentation Information Processing in Agriculture, In press, corrected proof, Available online 18 Information Processing in Agriculture, In press, corrected proof, Available online 18
November 2019

[16] D. Singh, N. Jain, P. Jain, P. Kayal, S. Kumawat, N. Batra. (2019). PlantDoc: A Dataset for Visual Plant Disease Detection.

[17] Tzutalin. 2015. LabelImg. Free Software: MIT License. https:// github. com/tzutalin/ labelImg

[18] Gonzalez R, Woods J. (2008). Digital image processing, 3rd edn. Prentice Hall, New Jersey.

[19] spMohanty/PlantVillage-Dataset, GitHub, 2019. Available: https:// github. com/ sp Mohanty/PlantVillageDataset/tree/master/raw/color/Tomato Late blight

[20] O. Hughes, D. Rugh,M. Salath' e. An open access repository of images on plant health to O. Hughes, D. Rugh,M. Salath e. An open access repository of im
enable the development of mobile disease diagnostics. ISA, 2015. 